\title{
Pattern recognition techniques for biological tissues differentiation in planktonic organisms
}

\author{
Morgado F.*, Posada N.G.**, Chavez M.G.*, Soares A.M.V.M.* and Lopez M.A.G.**
}

*CESAM \& Departamento de Biologia, Universidade de Aveiro, Campus Universitário de Santiago, 3810193 Aveiro, Portugal

*** Departamento de Electrónica, Universidade de Aveiro, Campus Universitário de Santiago, 3810-193 Aveiro, Portugal

email: fmorgado@ua.pt

Effective techniques for biological tissue differentiation and classification are critical in biological and ecological studies. Image data mining and sensing techniques have been developed and machine learning (ML) algorithms have been explored, however, tissue content differentiation is still a challenging problem. This work presents a novel method that appropriately combines image processing and ML techniques for content classification of biological tissues. The proposed method (Fig.1) comprises five steps: (1) computing the contour of organisms under study; (2) selecting representative samples of tissues and formed representative datasets of selected tissues; (3) training ML classifiers (i.e. artificial neural networks and support vector machines); (4) validating trained classifiers; and (5) classifying new tissues not use before in training.

A method prototype was implemented in MATLAB (R2013a). Figure 2 show a running example, from left to right and top to bottom: (1) original image; (2) gray level image; (3) resulting image after applying morphological gradient; (4) extracted tissue edges; (5) tissue classification: (5a) produced mask for vascular region, (5b) produced mask for gonadic cells region and (5c) produced mask for muscle region; (6) produced mask representing total tissue area; (7) vascular extracted tissue area; (8) gonadic cells extracted area; (9) muscle extracted tissue area; and (10) total tissue area.

The development and application of this computational method was a very important contribution to analyze and predict histological alterations. It allows to study positioning and distribution of cells and different cellular structures, qualitative evaluation of chemical contents, cellular or tissues morphological changes, using histological or histochemical data. This method was experimentally validated (on a representative image set of 200 planktonic organisms) both for biological tissues content differentiation and discerning the relevance of ecological parameters in the outcome of the quantification and prediction of organism's responses to human or natural disturbances.

[1] López, M. et al., Microscopy and Microanalysis, 19 (Suppl. 4), 75-76, 2013.

[2] Liang, J. et al., Medical Image Analysis, 10, 215-233, 2006.

[3] Moura D. and Lopez M., International Journal of Computer Assisted Radiology and Surgery, 8, 561-574, 2013. 
Development Workflow

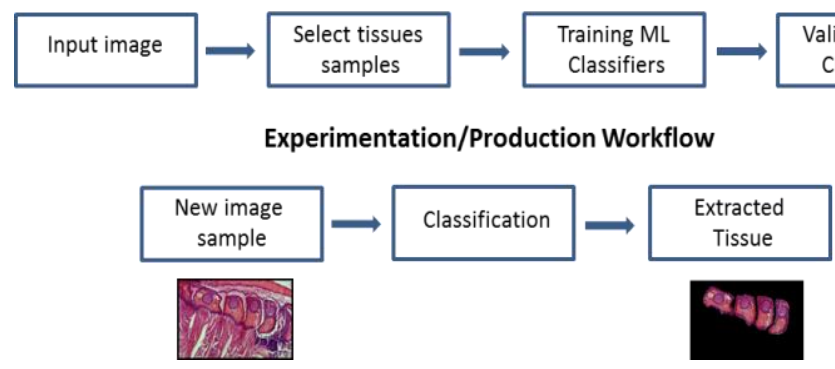
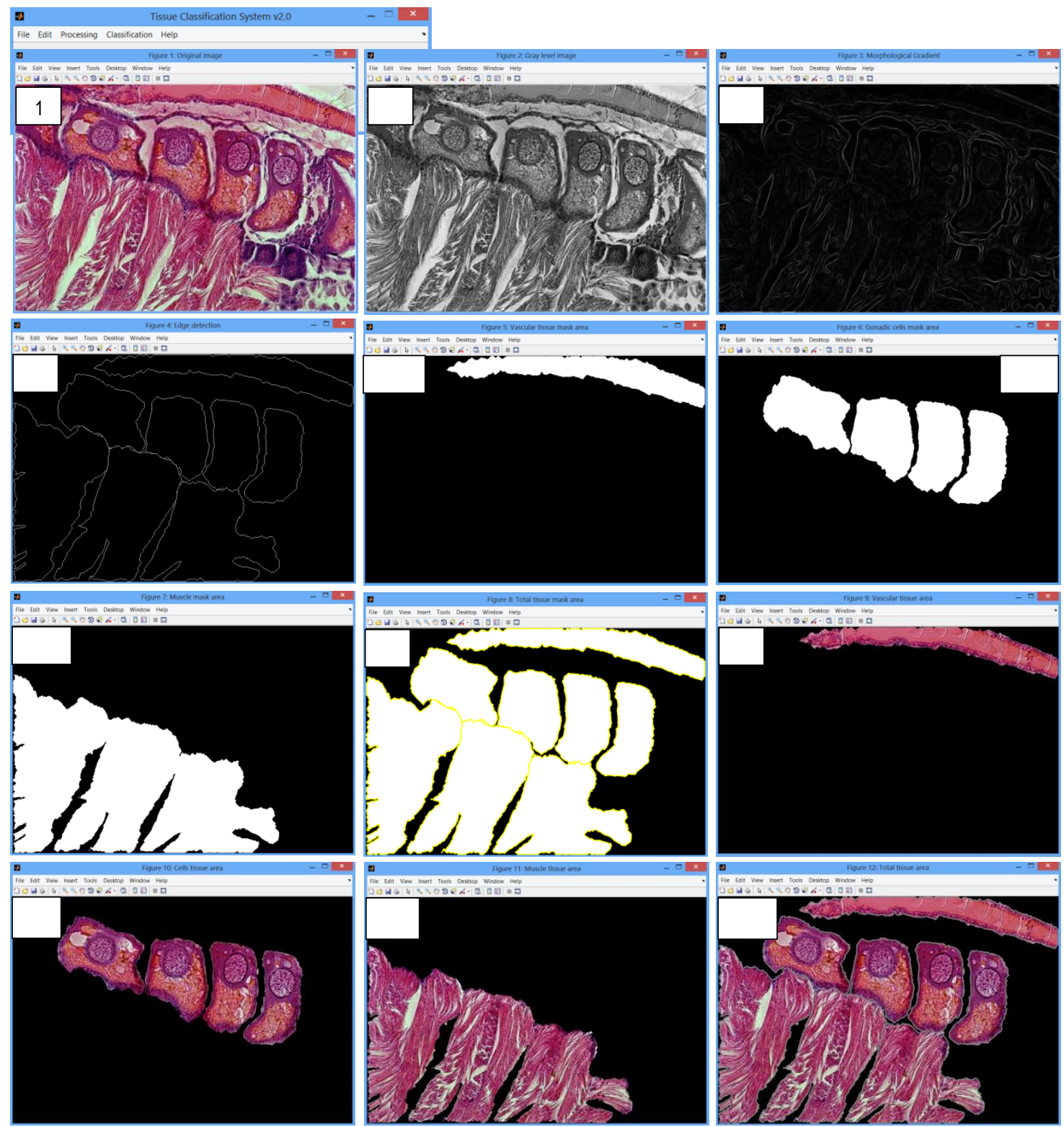

Fig.2. Tissue classification example using a method prototype developed in MATLAB. 